\title{
Direkte und indirekte Sprachgebrauchsstrategien beim Lösen von Französischaufgaben in der Grundschule
}

Léonie Fournier et Gérald Schlemminger

\section{(2) OpenEdition \\ Journals}

Édition électronique

URL : http://journals.openedition.org/esp/437

DOI : $10.4000 /$ esp.437

ISSN : 2532-0319

Éditeur

Centre d'Information sur l'Éducation Bilingue et Plurilingue

Édition imprimée

Date de publication : 1 décembre 2014

Pagination : 67-83

ISSN : 1127-266X

\section{Référence électronique}

Léonie Fournier und Gérald Schlemminger, « Direkte und indirekte Sprachgebrauchsstrategien beim Lösen von Französischaufgaben in der Grundschule ", Éducation et sociétés plurilingues [Online], 37 | 2014, Online erschienen am: 01 Oktober 2015, besucht am 01 Mai 2019. URL : http:// journals.openedition.org/esp/437 ; DOI : 10.4000/esp.437 


\section{DIREKTE UND INDIREKTE SPRAGHGEBRAUGHSSTRATEGIEN BEIM LÖSEN VON FRANZÖSISCHAUFGABEN IN DER GRUNDSCHULE}

\section{Léonie FourNiER, Gérald SchLEMMINGER}

Gli alunni delle classi bilingui di una scuola primaria franco-tedesca $\left(3^{\circ} \mathrm{e} 4^{\circ}\right.$ anno francese-tedesco) seguono alcune discipline insegnate in lingua 2 (DEL 2). Alla scuola primaria sono in immersione nella lingua di arrivo: questo permette loro di sviluppare numerose strategie al fine di comprendere meglio il contenuto delle varie materie. Eppure un buon numero di genitori si interroga: quali motivi fanno si che mio figlio sia in grado di capire il contenuto disciplinare in L2 senza passare per la verbalizzazione in L1? Com'è possibile che mio figlio parli questa lingua senza passare prima per l'apprendimento delle regole grammaticali? In quest'articolo presenteremo, argomenteremo ed analizzeremo le strategie di uso della lingua messe in atto da alunni della classe osservata per due anni scolastici.

Les élèves des classes bilingues d'une école primaire allemande ( $3^{\ell}$ et $4^{\ell}$ année français-allemand) suivent des disciplines enseignées en langue 2 (DEL 2). À l'école primaire, ils sont immergés en langue cible. Ce bain linguistique leur permet de développer de nombreuses stratégies afin de mieux appréhender le contenu des différentes matières. Cependant, nombre de parents se questionnent. Quelles raisons font que mon enfant puisse comprendre le contenu disciplinaire en L2 sans passer par la verbalisation en L1? Comment se fait-il que mon enfant parle cette langue sans passer d'abord par l'apprentissage des règles grammaticales?' Dans cet article, nous présenterons, argumenterons et analyserons les strategies spontanées d'usage de langue mises en place par les élèves de la classe observée durant deux années scolaires.

This article reports on an empirical study of $3^{\text {rd }}$ and $4^{\text {th }}$ grade students in a German-French bilingual school. The study consisted of research subjects solving exercises by using the think aloud method, after which film and transcription analysis allowed for a detailed examination of the strategies used during the exercises. Such strategies have been termed "language usage strategies" and have been distinguished from learning and communication strategies. This distinction is important because when students use their L2 in this context, their goal is not to learn the language or to communicate, but to solve the exercise. Individual findings revealed that the students manifested only a small number of language usage strategies in interviews concerning their L2 language habits, suggesting that they are not conscious of strategy usage when using their L2.

FRANZÖSISCHLERNE

N IM BILINGUALEN

SACHFACHUNTERRI

CHT IN DER

GRUNDSCHULE
Tn einigen deutschen Grundschulen lernen die SchülerInnen die Fremdsprachen immersiv. Dies bedeutet, dass sie in ein „Sprachbad" eintauchen und sich die Sprache über die Vermittlung von Sachfachinhalten aneignen. Die Sachfächer (meist Sport, Bildende Kunst, Textiles Werken, Sachkunde, 
Direkte und indirekte Sprachgebrauchsstrategien beim Lösen von

Französischaufgaben in der Grundschule

L. FOURNIER,

G. SCHLEMMINGER
Musik...) werden hierbei in der Zielsprache unterrichtet. Es wird jedoch nicht angestrebt, dass die Lernenden alle Inhalte immer im Detail verstehen. Was vor allem zählt, ist das globale Verstehen der Aussagen und der Handlungsanweisungen. Einstellungen zu Sprachlernprozessen

Viele Eltern fragen sich: Wie ist es möglich, dass mein Kind die Inhalte der Sachfächer in dieser Sprache versteht und auch wiedergeben kann, ohne eine genaue deutsche Übersetzung der Wörter zu erhalten und auch ohne zuvor grammatikalische Regeln gelernt zu haben? Wie schafft es mein Kind, die Sprache anzuwenden, Sätze zu erlesen und Fragen zu beantworten? Vor diesem Hintergrund haben wir uns im Rahmen einer empirischen Untersuchung (1) mit der Fragestellung beschäftigt, welche Denkprozesse in den Köpfen von SchülerInnen ablaufen, damit sie die Aussagen und Arbeitsaufträge der Lehrkräfte erfassen sowie Aufgaben lösen können.

In der Untersuchung wurden Dritt- und ViertklässlerInnen einer Grundschule mit bilingualem deutsch-französischem Zug in etwa fünf- bis zehnminütigen Interviews zu ihren Sprachgewohnheiten befragt. Die SchülerInnen schilderten auf Deutsch, wie sie sich verhalten, wenn sie die französischen Aussagen und Fragen des Lehrers nicht verstehen, wie sie reagieren, wenn sie Wörter nicht erlesen können und auch was sie tun, wenn sie nicht wissen, wie sie ein französisches Wort schreiben sollen.

Sie äußerten, dass:

- sie ihre Lehrkraft und MitschülerInnen um Hilfe bitten, wenn sie sich selbst nicht zu helfen wissen,

- sie in (Online-) Wörterbüchern nach der Übersetzung der Wörter nachsehen,

- sie einfach raten, was die Wörter bedeuten könnten.

Diese Aussagen zeigen, dass die SchülerInnen sich sehr allgemein äußern und sie sich ihrer Strategien kaum bewusst sind.

In unserer Untersuchung haben wir nun ein methodisches Vorgehen entwickelt, um besser zu verstehen, welche mentalen Prozesse beim Lösen von Aufgaben in der Zielsprache Französisch ablaufen und wie man diese Prozesse fördern kann. 
Direkte und indirekte Sprachgebrauchsstrategien beim Lösen von

Französischaufgaben

in der Grundschule

\section{FOURNIER,}

G. SCHLEMMINGER

STRATEGIEN BEIM LERNEN DER L2
Zahlreiche wissenschaftliche Studien des Fremdsprachenlernens zeigen, dass die SchülerInnen eine Reihe von Lernstrategien anwenden, wobei "[e]ine Lernstrategie [...] ein Plan (mentalen) Handelns [ist], um ein Ziel zu erreichen" (Bimmel 1993 zitiert nach Bimmel \& Rampillon 2000). Lernstrategien beinhalten meist Lerntechniken, also Fertigkeiten, die Lernende einsetzen, um ein Ziel zu erreichen. P. Bimmel und U. Rampillon (2000: 54f) nennen hierfür das Beispiel des Nachschlagens im Wörterbuch. Nur wer die Fertigkeit des Nachschlagens beherrscht, kann das Ziel (hier die Bedeutung eines Begriffs zu erfahren) erreichen.

Für die Lernenden bedeutet es, dass sie sich zuerst über das Ziel einer Aufgabe bewusst werden und dieses Ziel ggf. noch einmal formulieren müssen. Im Anschluss daran bauen sie einen (mentalen) Handlungsplan auf, um dieses Ziel zu erreichen. Schließlich führen sie den Plan durch konkretes Handeln aus.

Einige FremdsprachenforscherInnen (z.B. Oxford 1990: 14-21) unterscheiden zwischen direkten und indirekten Kategorien von Lernstrategien:

Direkte Sprachlernstrategien befassen sich unmittelbar mit dem Lernstoff. Sie bestehen wiederum aus:

- kognitiven Strategien, die dabei helfen, das neu Gelernte zu verstehen und zu bearbeiten,

- Gedächtnisspeicherstrategien, die hilfreich sind, das neu Gelernte abzuspeichern und zu einem späteren Zeitpunkt abzurufen, und

- Kompensationsstrategien, die den Lernenden helfen, ggf. Wissens- oder Informationslücken zu überbrücken.

Indirekte Sprachlernstrategien unterstützen den Lernprozess und schaffen Voraussetzungen, das eigene Lernen besser zu organisieren. Sie bestehen ihrerseits aus:

- metakognitiven Strategien, die über den Lernprozess selbst reflektieren,

- affektiven Strategien, die die Emotionen der Lernenden regulieren und

- sozialen Strategien, die die interpersonelle Kooperation unterstützen. 
Direkte und indirekte Sprachgebrauchsstrategien beim Lösen von

Französischaufgaben in der Grundschule

L. FOURNIER,

G. SCHLEMMINGER
P. Bimmel und U. Rampillon unterscheiden auch direkte und indirekte Sprachlernstrategien, wobei sie die Kompensationsstrategien nicht als direkte Sprachlernstrategie einordnen. Sie haben eine separate Kategorie gebildet, die als Sprachgebrauchsstrategie bezeichnet wird, die "nicht so sehr zum Erwerb einer Fremdsprache, sondern vielmehr beim kommunikativen Gebrauch der Fremdsprache angewandt" wird (Bimmel \& Rampillon 2000: 74-75). Diese Sprachgebrauchsstrategien werden oft zur Kompensation der Wissenslücken in schriftlichen und mündlichen Äußerungen genutzt. Sie beinhalten das "intelligente Raten", welches das Miteinbeziehen von linguistischen Anhaltspunkten, sprachlichen Hinweisen und das Ableiten von Bedeutungen aus dem Kontext erfordert. Deshalb betrachtet R. Oxford diese Strategien als Kompensationsstrategien, also als Teil der direkten Lernstrategien und führt sie nicht als Extrakategorie auf, wie Bimmel und Rampillon. Doch Sprachgebrauchsstrategien werden auch oftmals mit Kommunikationsstrategien gleichgesetzt:

En effet, dans ce contexte, on peut considérer que les stratégies d'usage seront en général, mais pas seulement, activées en situation de communication, et l'on peut alors différencier les situations de compréhension et de production, alors que les stratégies d'apprentissages seront en général, mais pas seulement, activées en dehors de ces situations, dans les phases d'étude et de préparation (Jeannot \& Chanier 2008: 7).

Wir sind der Meinung, dass zwischen Kommunikationsstrategien (stratégies de communication) und Sprachgebrauchsstrategien (stratégies d'usage) unterschieden werden muss, da die letzteren auch in Situationen benutzt werden, in denen nicht die Kommunikation das Ziel ist, wie z.B. beim Entnehmen von Informationen aus einem Sachtext, wo die Sprache lediglich einen instrumentellen Charakter hat. Sprachgebrauchsstrategien sind unseres Erachtens eine separate Kategorie von Strategien, die sich zwischen den Sprachlernstrategien und den Kommunikationsstrategien ansiedelt. Vor allem im bilingualen Sachfachunterricht, in dem Inhalte in der Fremdsprache vermittelt, angeeignet und verarbeitet werden, benutzen die Lernenden häufig diese Art von Strategien, bei denen weder das Lernen der Sprache noch die Kommunikation das erstrangige Ziel sind. 
Direkte und indirekte

Sprachgebrauchsstrategien beim Lösen von

Französischaufgaben

in der Grundschule

L. FOURNIER,

G. SCHLEMMingeR
Hauptsächlich der Gebrauch, also die konkrete Verwendung der Sprache zum Lösen einer Aufgabe ist hier die Absicht. Die Anwendung der Sprachgebrauchsstrategien findet (wie in den Transkriptionsausschnitten 1-5 zu sehen ist) meist in der L2 statt. Die Schüler sind sich (wie unsere Interviews gezeigt haben) über den häufigen und vielfältigen Einsatz ihrer Sprachgebrauchsstrategien kaum bewusst.

Direkte und indirekte Strategien kommen, sobald man ein Handlungsziel anstrebt und dieses mittels eines Planes erreichen möchte, in allen drei Situationen (Lernen - konkrete Verwendung der Sprache zum Lösen einer Aufgabe - Kommunikation) zum Einsatz. Demnach sollte man nicht nur von direkten und indirekten Sprachlernstrategien sprechen, sondern auch von direkten und indirekten Sprachgebrauchs- und Kommunikationsstrategien.

Die folgende Übersicht zeigt unser Verständnis von Strategien:

\begin{tabular}{|l|l|l|l|}
\hline & $\begin{array}{l}\text { Sprachlern- } \\
\text { strategicn }\end{array}$ & $\begin{array}{l}\text { Sprachgchrauchs- } \\
\text { strategicn }\end{array}$ & $\begin{array}{l}\text { Kommunikations- } \\
\text { strategicn }\end{array}$ \\
\hline Situation & $\begin{array}{l}\text { Extensiver, "klassischer" } \\
\text { Fremdsprachenunterricht }\end{array}$ & $\begin{array}{l}\text { Sachfachunterricht in der } \\
\text { Zielsprache oder intensiver } \\
\text { Fremdsprachenunterricht } \\
\text { an bilingualen Schulen }\end{array}$ & $\begin{array}{l}\text { Sprachenlernen in } \\
\text { authentischen Situationen } \\
\text { Bsp. Treffen mit } \\
\text { Austauschpartnern) }\end{array}$ \\
\hline Ziel & Sprache'n' lernen & $\begin{array}{l}\text { Gebrauch der Sprache } \\
\text { beim Lrwerben von } \\
\text { Inhalten des Sachfaches } \\
\text { oder der L2 Bsp: Lösen } \\
\text { von Aufgaben) }\end{array}$ & $\begin{array}{l}\text { Mitteilungen überbringen } \\
\text { und empfangen }\end{array}$ \\
\hline $\begin{array}{l}\text { LI oder L2 beim } \\
\text { Anwerden der } \\
\text { Strategien }\end{array}$ & meist L1 & meist L2 & $\begin{array}{l}\text { mcist code switching } \\
\text { zwischen L1 und L2 }\end{array}$ \\
\hline $\begin{array}{l}\text { Bewzistheitsgrad } \\
\text { schr bewusst }\end{array}$ & kaum unbewusst & bewusst \\
\hline
\end{tabular}

Tabelle 1: Typologie der Strategien 
Direkte und indirekte

Sprachgebrauchsstrategien

beim Lösen von

Französischaufgaben

in der Grundschule

L. FOURNIER,

G. SCHLEMMINGER

LAUTES DENKEN ALS FORSCHUNGSMETH ODE
In der zuvor erwähnten Untersuchung beobachteten wir die Sprachgebrauchsstrategien, die GrundschülerInnen beim Lösen von Aufgaben, die hier als Ziel das Verständnis der französischen Sprache haben, anwenden. Dazu benutzen wir als Forschungsinstrument das Laute Denken (Think aloud protocols). Diese introspektive Methode, auch als Selbst-Beobachtung bekannt, stammt aus der Psychologie und wird seit J. Corder (1981) in der Linguistik, besonders in der Sprachlehr- und lernforschung zur Elizitierung von verbalen Daten benutzt: "Simultane Introspektion findet in der Form des gleichzeitigen lauten Denkens oder der Verbalisierung spezifischer Aspekte wie z.B. Lese-, Schreib-, und Hörverstehensprozesse, Übersetzen und das Lösen schriftlicher Tests statt" (Kim 2006: 119).

Wir benutzen diese Methode in zweifacher Hinsicht:

a) als Datenerhebungsinstrument, um zu erforschen, über welche metalinguistische Reflexion die SchülerInnen in der Zielsprache verfügen und in welcher Sprache sie - wenn ihnen die Wahl freigestellt ist - denken, wenn sie Aufgaben in der Zielsprache lösen,

b) als didaktische Methode, um Bearbeitungsprozesse der Lernenden beim Lösen von Aufgaben (aller Art) zu vereinfachen und zu verbessern.

Auf methodischer Ebene zur Elizitierung von Laut-DenkProtokollen plädieren L. Heine und K. Schramm (2007: 178f) für eine Aufwärm- oder Übungsphase, in der die SchülerInnen an diese Technik herangeführt werden. Dabei sollten die Aufwärmübungen den anschließenden Lautdenkaufgaben jedoch wenig ähneln, da es sich um eine methodische und nicht um eine inhaltliche Vorbereitung handelt. Die meisten SchülerInnen sind es bekanntlich in ihrem Schulalltag beim Bearbeiten von Aufgaben hauptsächlich gewöhnt, leise zu denken. Folglich kommt es den Lernenden merkwürdig vor, ihre Gedanken laut zu äußern, wobei manche eingeschüchtert oder sogar gehemmt wirken und sich zunächst nicht trauen, laut zu sprechen. Aus diesem Grund müssen die Lehrkräfte ihre SchülerInnen zum Lauten Denken anleiten. 
Direkte und indirekte Sprachgebrauchsstrategien beim Lösen von

Französischaufgaben in der Grundschule

\section{FOURNIER,}

G. SCHLEMMINGER

\section{LAUTES DENKEN BEIM LÖSEN VON AUFGABEN IN DER ZIELSPRACHE}

BEISPIEL 1
Die SchülerInnen lösten im Rahmen unserer Langzeituntersuchung Französischaufgaben lautdenkend. Zuvor hatten sie ein Jahr lang täglich non-verbale Aufgaben bearbeitet. Dabei wurden unter anderem einfache Sudokus (4x4 Felder) und Mathematikaufgaben benutzt. Die Schüler mussten hierbei meist Elemente verbinden, umkreisen oder unterstreichen. Ziel dieser Übungsphase war das Trainieren der Technik, was dem einen oder anderen zu Beginn noch schwer fiel. Entweder wurde im Plenum am Tageslichtprojektor, im Tandem oder aber allein geübt. In der Durchführungsphase der wissenschaftlichen Studie bearbeiteten die SchülerInnen dann Aufgaben der Évaluations nationales des acquis des élèves en CE1 - Français (Ministère de l'éducation Nationale 2010 und 2011) (2). Diese werden in folgende Bereiche unterteilt: Lesekompetenz, Schreibkompetenz und die Sprachkunde, bestehend aus Wortschatz, Grammatik und Rechtschreibung. Wir entschieden uns dazu, Aufgaben aus folgenden Bereichen zu nehmen: lire, vocabulaire und grammaire. Beim Bearbeiten wurden die SchülerInnen gefilmt und im Anschluss von einer Lehrkraft interviewt.

Im Folgenden (Tabelle 2) erscheint eine erste Aufgabe aus dem Bereich vocabulaire:

Pour chaque mot de la première colonne, écris un mot de la même famille (Liste de mots: dent, nager, courir, beauté, chanter) Tabelle 2: Aufgabenbeispiel aus dem Wortschatzbereich

Der nun folgende Transkriptionsausschnitt Nr. 1 zeigt die Bearbeitung dieser Aufgabe durch einen Viertklässler. Ziel dieser Sprachaufgabe ist es, die Bedeutung eines Wortes, das gelesen wurde, zu verstehen und daraus ein weiteres Wort dieser Wortfamilie (egal welcher Wortart) zu bilden. 


\begin{tabular}{|l|l|l|l|l|}
\hline Turn & Zeit & Versuchsleiterin & SchülerIn & Extraverbal \\
\hline 65 & $07: 48$ & & $\begin{array}{l}\text { Dent, *le dent, qui fait un } \\
\text { travail avec *le dent? Le } \\
\text { dentiste. Le den-tis-te. }\end{array}$ & S schreibt dentiste. \\
\hline 66 & $08: 01$ & & Na-ger, la na- (:) tation. & S schaut I fragend an. \\
\hline 67 & $08: 10$ & M-hm. & & S schreibt natation. \\
\hline 68 & $08: 12$ & & La na-ta-tion. & \\
\hline
\end{tabular}

Direkte und indirekte Sprachgebrauchsstrategien beim Lösen von Französischaufgaben in der Grundschule

L. FOURNIER,

G. SCHLEMmingeR
Transkriptionsausschnitt 1 (FMB20121204 T4 10;1)

Der Schüler stellt sich im Turn 65 selbst eine Frage, indem er nach dem handelnden Subjekt fragt: „Dent, *le dent, qui fait un travail avec *le dent?" Er beantwortet diese an sich selbst adressierte Frage mit "Le dentiste". Im Turn 66 spricht er das Wort nager langsam, in getrennten Silben aus, und nennt nach kurzem, stillem Denken das Wort natation. Anschließend schaut er den Interviewer kurz fragend an, um eine (Selbst-) Bestätigung für das Gesagte zu bekommen, bevor er mit dem Schreiben des Wortes beginnt. Der Schüler spricht beim Schreiben die Silben der Wörter jeweils langsam laut aus: Le den-tis-te im Turn 65 und La nata-tion im Turn 68.

Der Schüler analysiert den Satz im Turn 65, indem er die im Muttersprachenunterricht erlernte Regel der Frage nach dem Subjekt des Satzes „Wer oder was tut etwas?“ zielsprachig richtig anwendet. Das Fragen nach dem Subjekt gehört zu den kognitiven Strategien. Wir zählen sie zu den direkten Sprachgebrauchsstrategien.

Im Turn 66 erkennt der Schüler vermutlich durch das Betonen der Anfangssilbe "na", dass er daraus ein neues Wort derselben Wortfamilie bilden kann. Das Ableiten und Herleiten von Wörtern ist eine kognitive Strategie, die besonders bei zusammengesetzten und abgeleiteten Wörtern ihre Anwendung findet. "Der Lernende findet die Bedeutung neuer Ausdrücke heraus, indem er sie zerlegt" (Bimmel / Rampillon 2000: 69). Wir zählen diese Strategie ebenfalls zu den direkten Sprachgebrauchsstrategien. 
Direkte und indirekte

Sprachgebrauchsstrategien beim Lösen von

Französischaufgaben

in der Grundschule

L. FOURNIER,

G. SCHLEMMINGER

BEISPIEL 2
Das (bewusste) Benutzen von einzelnen Lauten oder Silben ist unser Ansicht nach auch oft eine Gedächtnisstrategie, die dazu dient, sich Wörter im Arbeitsgedächtnis abzuspeichern, um sie dann sofort wieder abrufen zu können. Im Turn 65 und 68 helfen die Silbentrennungen dem Schüler zudem bei der korrekten Orthographie dieser neugebildeten Wörter. Wir würden diese Strategie auch zu den direkten Sprachgebrauchsstrategien zählen.

Bei der nonverbalen Handlung „S schaut I fragend an” handelt es sich nicht um eine soziale Strategie, da der Schüler keine direkte Frage an den Interviewer stellt und auch nicht um Korrektur bittet. Es handelt sich eher um eine affektive Strategie, da er sich durch den Blickkontakt zu der Versuchsleiterin selbst bestärkt hat. Diese Selbstbestätigung ist eine Form der positiven Bestärkung und der eigenen Motivierung. Sie gehört somit zu den indirekten Sprachgebrauchsstrategien.

In Klassenarbeiten wird das Laute Denken beim Schreiben von Wörtern und Sätzen meistens leider nicht gestattet, da es andere Schüler ablenken und ihnen außerdem die richtige oder falsche Lösung geben würde. Angesichts der hier angeführten Beispiele sollten die Lehrkräfte das lautdenkende Bearbeiten von Aufgaben jedoch überdenken.

Hier nun ein zweites Beispiel aus dem grammatikalischen Bereich:

Range les mots soulignés dans les colonnes Noms; Articles; Verbes.

Paul aime les gâteaux. La petite fille lui propose une part énorme de tarte. Il demande un autre morceau.

Tabelle 3 Aufgabenbeispiel aus dem grammatikalischem Bereich 


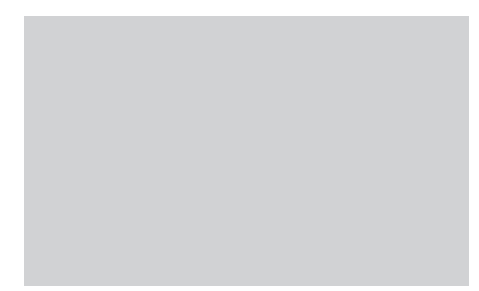

Der folgende kurze Transkriptionsausschnitt Nr. 2 zeigt den Anfang und das Ende der Bearbeitung dieser Aufgabe.

\begin{tabular}{|l|l|l|l|l}
\hline Turn & Zeit & $\begin{array}{l}\text { Versuchsleiteri } \\
\mathrm{n}\end{array}$ & SchülerIn & Extraverbal \\
\hline 161 & $25: 30$ & & $\begin{array}{l}\text { Le caramel bouillant (undeutlich } \\
\text { ausgesprochen) colle a la langue. } \\
\text { Ahm, Souligne le verbe, ahm, le verbe. } \\
\text { Qu'est-ce qu'il fait? Il colle. }\end{array}$ & $\begin{array}{l}\text { S unterstreicht } \\
\text { colle. }\end{array}$ \\
\hline 162 & $25: 43$ & $\begin{array}{l}\text { Et qui colle? Le caramel *huyant } \\
\text { [byjä]. } \\
\text { Ou juste le caramel? Je *sais pas. } \\
\text { Mais je fais le caramel *buyant } \\
\text { byjäl. Oui. }\end{array}$ & $\begin{array}{l}\text { S umkreist Le } \\
\text { caramel bouillant. }\end{array}$ \\
\hline
\end{tabular}

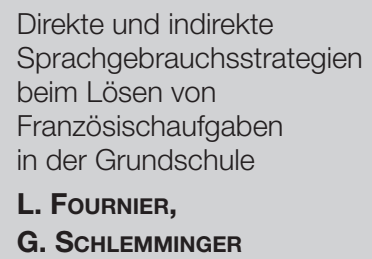

G. SCHLEMMINGER

Transkriptionsausschnitt 1 MMJ20121214 T4 9;5

Zu Beginn (im Turn 93) behauptet der Schüler: „[Die Aufgabe] ist ja leicht". Trotzdem stockt der Schüler, da er sich offensichtlich nicht sicher ist, zu welcher Wortart aime gehört. Er geht zu dem zweiten Wort, einem (täglich verwendeten) Artikel und dann zum dritten Wort, einem (bekannten) Nomen, über. Das Wort aime liest er in der Bearbeitung (Turn 105) noch einmal und ordnet es richtig als Verb zu. Am Ende der Aufgabe (Turn 111) überprüft der Schüler schließlich durch Abzählen "Un, deux, trois [...]", ob er alle unterstrichenen Wörter aus den Beispielssätzen in die Tabelle eingetragen hat und ob er keine ausgelassen hat.

Im Turn 93 bestärkt sich der Schüler selbst, indem er sich Mut zuredet. Dies ist eine affektive Strategie, die wir zu den indirekten Sprachgebrauchsstrategien zählen.

Bei diesem Schüler stellen wir fest, dass er dieses Wort überspringt, sobald er bemerkt, dass er bei der Wortgruppenzuordnung an einer Stelle nicht voran kommt und sich zunächst einem anderen Aspekt der Aufgabe widmet; er kommt dann später nochmals auf den vorigen Aspekt zurück. Der Plan und dessen Ausführung laufen hier nicht wie vorhergesehen ab. Sich einem neuen Teilaspekt einer Aufgabe zu widmen, verstehen wir als Regulierung des eige- 


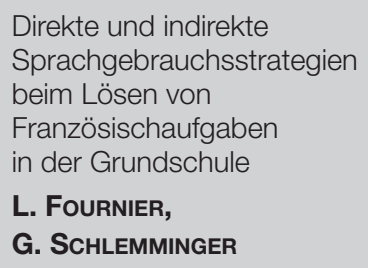

BEISPIEL 3

\begin{tabular}{|l|l|l|l|l|}
\hline Turn & Zeit & $\begin{array}{l}\text { Versuchsleiteri } \\
\mathrm{n}\end{array}$ & SchülerIn & Extraverbal \\
\hline 75 & $10: 09$ & & $\begin{array}{l}\text { Mal-heu-reux est (:) was ist das } \\
\text { Gegenteil davon? Fe crois heureux, } \\
\text { oui. }\end{array}$ & S schreibt heureux. \\
\hline 76 & $10: 28$ & & $\begin{array}{l}\text { Compliqué (:) hmm, was ist das } \\
\text { Gegenteil? (:) *ncompliqué? Non, } \\
\text { pas compliqué (:). Oui, je crois. }\end{array}$ & $\begin{array}{l}\text { S schreibt pas } \\
*^{*} \text { compliquer. }\end{array}$ \\
\hline 77 & $10: 58$ & & Ouvir (:) et fermer, c'est clair. & S scheibt *ermé. \\
\hline
\end{tabular}

nen Lernens, die bewusst gesteuert wird. Es ist eine metakognitive Strategie; sie zählt zu den indirekten Sprachgebrauchsstrategien. Das Überprüfen der Richtigkeit und Vollständigkeit einer Aufgabe (im Turn 111) ist ebenso eine metakognitive Sprachgebrauchsstrategie. Diese Strategie wird von Rebecca Oxford als self-monitoring bezeichnet. Bimmel und Rampillon benennen sie als "Strategie zur Regulierung des eigenen Lernens" $(2000,71)$. Sie sprechen von einem "Manager im Kopf", der die Aufgaben analysiert und sie dann plant, indem er realisierbare Ziele bestimmt und sie überprüft. Der Schüler hatte sich als Ziel gesetzt, alle Wörter den richtigen Wortarten zuzuordnen und überprüft dies am Ende nochmals. Diese indirekte Sprachgebrauchsstrategie ist sehr sinnvoll, wird aber von den SchülerInnen allgemein nur selten angewendet, da sie zeiteinnehmend ist.

Das Verbinden mehrere Strategien ist ein oft zu beobachtendes Vorgehen. Manchmal stellt sich eine Strategie als ungeeignet heraus und muss modifiziert werden, oder eine ganz andere Strategie muss an diese Stelle treten.

Hier nun ein Beispiel aus dem Bereich des Wortschatzes:

Écris un mot qui veut dire le contraire de chaque mot: malheureux; compliqué; ouvrir; reculer; rapide; intérieur.

Tabelle 4 Aufgabenbeispiel aus dem Wortschatzbereich

Der folgende Transkriptionsausschnitt Nr.3 zeigt die Vorgehensweise eines Schülers bei der Suche nach den Antonymen.

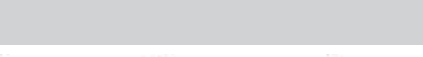


Direkte und indirekte Sprachgebrauchsstrategien beim Lösen von

Französischaufgaben

in der Grundschule

L. FOURNIER,

G. SCHLEMMINGER

BEISPIEL 4

\begin{tabular}{|l|l|l|l|l|}
\hline Turn & Zeit & $\begin{array}{l}\text { Versuchsleiter } \\
\text { in }\end{array}$ & SchülerIn & Extraverbal \\
\hline 93 & $13: 48$ & & $\begin{array}{l}\text { Ist ja leicht. 'Aime' est... non! } \\
\text { 'Les' c'est article. 'Gâteaux' } \\
\text { ''est le...un nom. }\end{array}$ & \\
\hline & & & $\ldots .$. & \\
\hline 105 & $16: 20$ & & $\begin{array}{l}\text { Aimer, alors aime. Aimer, das } \\
\text { tut man, also c'est un verbe. }\end{array}$ & $\begin{array}{l}\text { S schreibt aime zu } \\
\text { den Verben. }\end{array}$ \\
\hline 109 & $17: 02$ & & $\ldots$ & \\
\hline 110 & $17: 04$ & M-hm. & Oui. Je crois c'est tout. & \\
\hline 111 & $17: 05$ & & $\begin{array}{l}\text { Un, deux, trois, quatre, cinq, } \\
\text { six, sept, huit, neuf. Un, deux, } \\
\text { trois, quatre, cinq, six, sept, } \\
\text { huit, neuf. Oui. }\end{array}$ & $\begin{array}{l}\text { S zählt Anzahl der } \\
\text { Wörter nach. }\end{array}$ \\
\hline
\end{tabular}

Im Turn 68 übersetzt der Schüler das Wort ouvrir ins Deutsche, sucht dann das deutsche Gegenteil und übersetzt es anschließend wieder ins Französische. In Turn 72 und 73 geht er auf die gleiche Art und Weise vor, wobei hier eine kurze Pause eintritt, bevor er die deutsche Übersetzung der Wörter findet.

Das Übersetzen und der Vergleich mit der Muttersprache, bzw. das Rekurrieren auf die Kenntnisse in der L1 sind Teil von direkten, kognitiven Sprachgebrauchsstrategien. Die kleinen Pausen (Turn 72 und 73) zeigen unseres Erachtens, dass der Schüler kurz leise nachdenkt, um die Übersetzung der Wörter zu finden. Wir wissen nicht, wie er die Übersetzung gefunden hat, da er sich nicht laut dazu geäußert hat. Wir vermuten, dass es dem Schüler nicht gelingt, das Antonym direkt in der L2 zu benennen, weil für ihn die Aufgabe wohl zu abstrakt bleibt.

Der Transkriptionsausschnitt Nr. 4 zeigt bei derselben Aufgabe (Tabelle 4) die Vorgehensweise eines Schülers, der bei der Suche nach dem Antonym nicht auf die direkte deutsche Übersetzung zurückgreift. 
Direkte und indirekte Sprachgebrauchsstrategien beim Lösen von

Französischaufgaben in der Grundschule

\section{FOURNIER,}

G. SCHLEMmingeR

BEISPIELE 5

Der Schüler legt in den Turns 75, 76 und 77 nach dem Benennen des zu analysierenden Wortes eine Denkpause ein. Dann stellt er sich selbst in der Ll die Frage nach dem Antonym des Wortes. Er findet dann direkt das entsprechende französische Wort. Im Turn 75 leitet er die Benennung des Antonyms mit "je crois" ein, den Turn 76 beschließt er damit. Den Turn 77 beendet er mit "c'est clair". Im Turn 76 benutzt der Schüler zur Antonymbildung das Verfahren der Präfigierung, d.h., er stellt den gebundenen Negationspräfix ,,in-“ nach dem Modell ,,compréhensible - incompréhensible“ dem Adjektiv vor. Er scheint zu erkennen, dass das Derivationsschema in diesem Fall keine Anwendung findet („Non“) und setzt dem Adjektiv den Negationspartikel pas voraus: „pas compliqué“. Er schreibt das Wort nicht, wie zu erwarten gewesen wäre, als Adjektiv pas compliqué, sondern - dem Negationspartikel voll angemessen - als Verb infintiver Form nieder: „pas compliquer“. (Beim Niederschreiben des Antonyms von oworir in Turn 77 begeht er genau den entgegengesetzten Fehler, indem er die partizipiale Form des Verbs notiert.) Dieser komplexe Prozess der Sprachgenerierung zeigt ein hohes, ausgeprägtes Sprachsystembewusstsein dieses Schülers an.

Mit der prospektiven Äußerung ,je crois..." des Schülers tritt eine Subjektivierung des Diskurses ein. Wir vermuten, dass er sich bei der Ausführung der Aufgabe nicht ganz sicher ist, jedoch benötigt er nicht die Hilfe oder Bestätigung der Versuchsleiterin. Die Art und Weise, wie der Schüler seinen Diskurs subjektiviert, legt nahe, dass er sich hier Mut zuspricht. Es ist eine affektive Strategie innerhalb der indirekten Sprachgebrauchsstrategien. Der Schüler wechselt in diesem Ausschnitt mehrmals von der L2 zu L1. Dieses Codeswitching gehört zu den direkten, kognitiven Sprachgebrauchsstrategien.

Hier nun ein letztes Beispiel aus dem grammatikalischen Bereich.

Pour chaque phrase, souligne le verbe et entoure le sujet.

- Le gâteau était fameux.

- Il renifle un bon coup.

- Le caramel bouillant colle à la langue. 


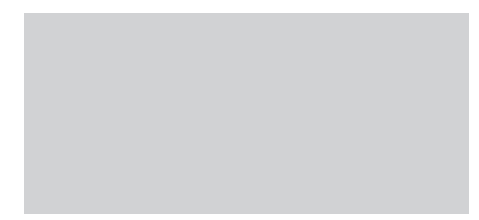

Der folgende kurze Transkriptionsausschnitt Nr. 5 zeigt den letzten Teil der Bearbeitung dieser Aufgabe.

\begin{tabular}{|l|l|l|l|l|}
\hline Turn & Zcit & $\begin{array}{l}\text { Versuchslcitcri } \\
\mathrm{n}\end{array}$ & SchülcrIn & Extraverbal \\
\hline 161 & $25: 30$ & & $\begin{array}{l}\text { Le caramel bouillant (undcutlich } \\
\text { ausgesprochen) colle à la langue. } \\
\text { Ahm, Souligne le verbe, ähm, le verbe. } \\
\text { Ou'est-ce qu'tl fait? Il colle. }\end{array}$ & $\begin{array}{l}\text { S unterstreicht } \\
\text { colle. }\end{array}$ \\
\hline 162 & $25: 43$ & & $\begin{array}{l}\text { Et qui colle? Le caramel *buyant } \\
\text { [byjã]. } \\
\text { Ou juste le caramel? Je * sais pas. } \\
\text { Mais je fais le caramel *buyant } \\
\text { [byjã]. Oui. }\end{array}$ & $\begin{array}{l}\text { S umkreist Le } \\
\text { caramel bouillant. }\end{array}$ \\
\hline
\end{tabular}

Direkte und indirekte Sprachgebrauchsstrategien beim Lösen von Französischaufgaben in der Grundschule

L. FOURNIER,

G. SCHLEMMINGeR
Transkriptionsausschnitt 5 (LFF20121210 T4 9;10)

Im Turn 161 liest die Schülerin den Satz und wiederholt die Aufgabenstellung: Sie fragt auf Französisch nach der Aktivität, also syntaktisch nach dem Verb: „..Qu'est-ce qu'il fait?" und beantwortet sich diese Frage, indem sie das richtige Verb nennt: „Il colle. " und dann gemäß der Aufgabenstellung das Verb unterstreicht.

Im Turn 162 fragt die Schülerin wieder auf Französisch nach dem Subjekt „Et qui colle?" und beantwortet sich die Frage, indem sie die ganze Nominalgruppe (Artikel + Nomen + Adjektiv) nennt: „Le caramel *buyant" Sie ist sich nicht sicher und fragt sich, ob nur Artikel und Nomen das Subjekt repräsentieren: „Ou juste le caramel?.“. Sie entscheidet, die ganze Nominalgruppe (Artikel + Nomen + Adjektiv) dem Subjekt zuzuordnen: „Mais je fais le caramel *buyant" (3). Vermutlich möchte die Schülerin mit dem häufig gebrauchten Verb faire in "Mais je fais [...]" ausdrücken, dass sie sich für diese Nominalgruppe entscheidet und diese umkreist. Das Nachdenken über Satzelemente ist eine kognitive Strategie und gehört zu den direkten Sprachgebrauchsstrategien.

Sie bekräftigt ihre Entscheidung mit einem affirmativen ,oui“ am Turnende, um ihre Lösung für richtig zu erklärten, und umkreist entsprechend der Aufgabe diese Nominalgruppe. 
Direkte und indirekte Sprachgebrauchsstrategien beim Lösen von

Französischaufgaben

in der Grundschule

L. FOURNIER,

G. SCHLEMmingeR

PÄDAGOGISCHE KONSEQUENZEN UND LEHRSTRATEGIEN
Sich Bestärken und auch sich selbst einschätzen ist eine affektive Strategie und ist den indirekten Sprachgebrauchsstrategien zuzuordnen.

Das Wiederholen einer Aufgabenstellung (Turn 161) ist eine metakognitive Strategie, hier vermutlich, um sich die Aufgabenstellung erneut deutlich zu machen; sie zählt zu den indirekten Strategien. Im Turn 162 fragt sie nach dem Verb. Dies ist vermutlich eine Strategie, die sie im Deutschunterricht gelernt hat. Sie überträgt also eine L1-Strategie auf die L2. Das Fragen nach dem Verb ist eine kognitive Strategie, die zu den direkten Sprachgebrauchsstrategien zählt.

Die Transkriptionsausschnitte zeigen, dass die Schüler und Schülerinnen über verschiedene direkte und indirekte Sprachgebrauchsstrategien verfügen, die sie allerdings kaum isoliert anwenden, sondern meist kombiniert, obwohl sie sich dieser nicht immer bewusst sind. Lediglich die Lerntechniken und einige soziale Strategien scheinen ihnen präsent zu sein. Doch das Bewusstsein über Strategien erleichtert die Anwendung dieser in konkreten Sprachgebrauchssituationen.

An Hand dieser Studie können wir zeigen, wie wichtig die Sprachgebrauchsstrategien zum Verstehen und Praktizieren der Sprache sind. Aus diesem Grund ist es spracherwerbstheoretisch sinnvoll, den Schülern diese Strategien auch bewusst zu machen. Auf der pädagogischen Ebene kann dies durch verschiedene Herangehensweisen geschehen. Wichtig ist dabei, dass nicht die Lehrkraft den SchülerInnen vorschreibt, wann und wie sie die Strategien anwenden sollen, sondern dass die SchülerInnen diese selbst entdecken. Die Lernenden sollen sie so anwenden, dass sie zum angestrebten Ziel (Lösung der Aufgabe usw.) führen.

Um die SchülerInnen ihre Sprachgebrauchsstrategien bewusst zu machen, bedarf es eines vorausgehenden, aufgaben- und lernerorientierten Trainings. Sowohl Oxford (1990) als auch Bimmel und Rampillon (2000) zeigen die Wichtigkeit des inhaltsverknüpften Strategielernens auf und betonen, dass inhaltsloses Lernen nicht zum gewünschten Erfolg führt. In der Grundschule sollte es unseres Erachtens 
Direkte und indirekte Sprachgebrauchsstrategien beim Lösen von

Französischaufgaben in der Grundschule

\section{FOURNIER,}

G. SCHLEMMINGER für den Klassenlehrer kein Problem darstellen, das "Lernen lernen" im Rahmen des Klassenlehrerunterrichts anzubieten und Inhalte aus den aktuell behandelten Themen mit einzubeziehen.

In der Klassensituation bedeutet dies, dass in einem ersten Schritt entweder die Lehrperson oder aber die SchülerInnen ihre Lautdenkprozesse ihren Klassenkameraden vorstellen, während sie eine Aufgabe bearbeiten. Dies kann beispielsweise an der Tafel oder am Tageslichtprojektor beim Lösen von Aufgaben stattfinden. In einem zweiten Schritt werden diese Strategien an konkreten Problemen der verschiedenen Unterrichtsfächer geübt. Zum Üben bieten sich besonders offene Aufgabenstellungen an, in denen es keine festgelegte Vorgehensweise gibt und die Schüler selbst experimentieren können, wie sie vorgehen und welche Strategien sie anwenden möchten. Dies kann alleine oder im Tandem geschehen. Bimmel und Rampillon unterstreichen, dass die Schüler sich im Tandem über ihre angewandten erfolgreichen Strategien austauschen sollten:

Die Arbeit in Zweiergruppen ist die Arbeitsform, die für reflektierende Aktivitäten am geeignetsten erscheint. Lernende, die zu zweit arbeiten, erzählen einander, wie sie bei der Lösung der Aufgabe vorgehen. Das ist erstens funktional, und zweitens kommt sich der einzelne Lernende dabei weniger dumm vor, als wenn er dasitzt und allein vor sich hinspricht, um zu verbalisieren, was er macht (Bimmel und Rampillon 2000: 94).

Doch auch über misslungene Strategien sollte diskutiert werden; es sollte nach der Ursache des Misserfolgs gesucht werden. In einem dritten Schritt bietet es sich an, gemeinsam mit den Schülern ein Strategien-Handbuch oder Poster zu erstellen, in dem es eine Übersicht der Strategien und zu den Beispielsaufgaben gibt. 


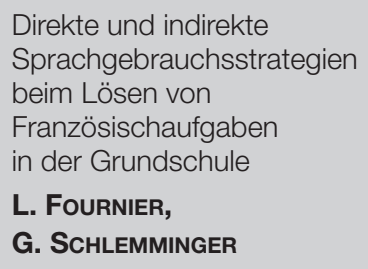

\section{LITERATURVER-} ZEICHNIS

BIMMEL P. 1993. Lernstrategien im Deutschunterricht. In: Fremdsprache Deutsch, H. 8/1993 "Lernstrategien", 4-11.

BIMMEL P. \& RAMPILLON U. 2000. Lernerautonomie und Lernstrategien. München: Langenscheid.

HEINE L. \& SCHRAMM K. 2007. Lautes Denken in der Fremdsprachenforschung. Eine Handreichung fir die empirische Praxis, pp. 167206 in H. J. VOLLMER (Hrsg.), Synergieeffekte in der Fremdsprachenforschung: Empirische Zugänge, Probleme, Ergebnisse. Frankfurt a.M.: Lang.

HEINE L. 2010. Problem solving in a foreign language. A study in content and language integrated learning. Berlin: Walter de Gruyter.

JEANNOT L. \& CHANIER T. 2008. Stratégies d'un apprenant de langue dans une formation en ligne sur une plate-forme audio-synchrone, in Apprentissage des Langues et Systèmes d'Information et de Communication (ALSIC), vol. 11.

KIM O.-S. 2006. Introspektion in der L2 Erwerbsforschung- Ein Beispiel des Bedeutungserschließungsprozesses durch lautes Denken, pp. 115-133 in Dogilmunhak Koreanische Zeitschrift fir Germanistik. Band 98, Jahrgang 47/ Heft 2.

Ministère de l'éducation nationale, de la jeunesse et de la vie associative. 2010. Evaluation nationale des acquis des élèves en CE1. Français et Mathématiques. Mai 2010. Cahier de l'élève. Portal des französischen Erziehungsministeriums: http://media.education.gouv.fr/file/evaluations/79/6/Cahier_eleve_CE1_2010_145796.pdf

Ministère de l'éducation nationale, de la jeunesse et de la vie associative. 2011. Evaluation nationale des acquis des élèves en CE1. Français et Mathématiques. Mai 2011. Cahier de l'élève. Portal des französischen Erziehungsministeriums: http://media.education.gouv.fr/file/evaluations/93/6/CE1_cahier_eleve_2011_180936.pdf

OXFORD R. 1990. Language Learning Strategies. What every teacher should know. Boston Massachussets: Heinle \& Heinle Publishers.

\section{NOTES}

(1) Die Untersuchung wird im Rahmen einer Langzeitstudie durchgeführt. Die Dissertation (Fournier) dieser Langzeitstudie ist in Bearbeitung.

(2) Wir benutzen einen französischen standardisierten nationalen Test der Grundschule, der aus kurzen geschlossenen Aufgaben besteht und somit die Auswertung erleichtert. Diese Tests waren bis zum Jahre 2012 für alle Grundschulen Frankreichs am Ende der 2. Klasse (CE1) verpflichtend.

(3) Der Aussprachefehler [byjã] statt [bujã] mag sich eventuell daher erklären, dass dieses Wort der Schülerin nicht sehr geläufig, ggf. gar unbekannt ist. 